\title{
Robust Approximations to the Distribution of Link Distances in a Wireless Network Occupying a Rectangular Region
}

\author{
John P. Mullen, Ph.D \\ jomullen@nmsu.edu \\ Industrial Engineering Department \\ New Mexico State University
}

\begin{abstract}
An important characteristic of a Mobile Ad Hoc Network (MANET) is the distribution of the link distance between communicating nodes. Knowing this distribution is a step in determining the distribution of the number of neighbor nodes, the number of hops required to transmit a message, etc. This paper presents two robust approximations to the link distance distribution for nodes within a rectangular area, providing the $x$ and $y$ node coordinates follow independent continuous spatial distributions. This paper also presents an approximate link distance distribution for the Random Waypoint Mobility model and examines the approximation error in a number of situations.
\end{abstract}

\section{Introduction}

An important characteristic of a Mobile Ad Hoc Network (MANET) is the distribution of the link distance between communicating nodes. This distribution is needed to determine the distribution of the number of hops required to complete a transmission, the number of neighbor nodes, etc. It is also needed to calculate probabilities, such as that of having at least one neighbor or of a network partition.

The link distance distribution is a consequence of the spatial distribution of the nodes. One may derive this distribution on a case-by-case basis, but the process can be quite tedious, time consuming, and error-prone. Also, the different resulting models of the link length distribution complicate the comparison of different situations.

This paper presents two robust parametric models of the link distance distribution which are appropriate in a large number of cases, including the Random Waypoint Mobility Model. It also demonstrates their use and states the resultant approximation error in a number of situations.

\section{I.A. Background}

This paper was primarily inspired by a recent paper in which the author derived an approximate link distance distribution which seemed to work well when nodes are uniformly distributed. However, the author presented little to explain the phenomena, speculating that the underlying spatial distribution may not be very important [1]. This paper explores the reasons such approximations work and demonstrates that certain aspects of the underlying spatial distribution are very important, while others can be ignored. It also presents two improved versions of the original estimator.

\footnotetext{
*This work is supported by the U.S. Army Training and Doctrine Command Analysis Center (TRAC) and New Mexico State University. (c) ACM, 2003. This is the author's version of the work. It is posted here by permission of ACM for your personal use. Not for redistribution. The definitive version was published in Mobile Computing and Communications Review, 7 (2) (April, 2003) http://doi.acm.org/10.1145/960000/950393/p80-mullen.pdf
}

A second inspiration was a recent study of nodes in motion in the Random Waypoint Mobility Model. The authors demonstrated that the spatial distribution is not uniform and presented a plausible distribution for nodes in constant motion [2]. Using their results, this paper develops a more general approximate distribution of link distances when the Waypoint model is used.

\section{I.B. Overview}

Assumptions and notation are stated in the remainder of this section. Section II of this paper outlines a straightforward method to approximate the link distance distribution, given the spatial distribution of points. Section III develops an approximate link distance distribution in the Waypoint model as a function of the probability that a communicating node is in motion. In Section IV, the nature of the approximation error is examined in the general case. Section V summarizes, draws conclusions and suggests future work.

Each key point is illustrated with examples, three of which are directly related to the Waypoint model. The body of the paper deals with results and applications. The Appendices present derivations of those results.

\section{I.C. Assumptions}

To streamline the presentation, assume that nodes are distributed according to some continuous bivariate distribution over a $w$ by $h$ rectangle. Also assume a node's $x$ and $y$ coordinates are chosen independently. Finally, assume the members of a node pair are chosen independently.

These assumptions are consistent with those made in the Waypoint model. In addition, as noted later, some of these assumptions may be relaxed.

\section{I.D. Notation}

This paper discusses a number of different random variables and distribution functions. In an attempt to retain order, the following conventions are used. Random variables are denoted by upper case letters and specific instances of 
those random variables by lower case letters. The density function of a random variable is denoted by a lowercase " $f$ " subscripted with the label of the random variable. Hence $f_{X}(x)$ denotes the value of the density function for the random variable $X$ at point $x$.

For each situation, there are three principal distributions.

1. The spatial distribution, $f_{X, Y}(x, y)$, is the joint probability density function for the $x$ and $y$ coordinates of a randomly selected node.

2. The difference distribution, $f_{\vec{\Delta}}\left(\delta_{X}, \delta_{Y}\right)$, is the joint probability density function for the differences $\Delta_{X}=$ $X_{1}-X_{2}$ and $\Delta_{y}=Y_{1}-Y_{2}$ for two randomly-selected nodes, $N_{1}=\left(X_{1}, Y_{1}\right)$ and $N_{2}=\left(X_{2}, Y_{2}\right)$. This may also be stated in polar coordinates as $f_{\vec{\Delta}}(r, \theta)$.

3. The link distance distribution, $f_{R}(r)$, is the probability density function for $R=\sqrt{\Delta_{X}^{2}+\Delta_{Y}^{2}}$, the link distance between two randomly-selected communicating nodes.

Each basic function may have several forms. The cumulative distribution function (CDF) will be denoted as a subscripted " $F$." Approximations to a probability density or cumulative distribution function will be denoted " $g_{v}$ " and " $G_{v}$ ", respectively. Here, " $v$ " refers to the specific version of the estimate.

The examples include five underlying distributions. The letter in the list below will be used as a subscript to distinguish among them.

$U$ The uniform spatial distribution, for which the exact distribution of $R$ is given in [1].

M A spatial distribution of nodes in constant random motion based on that in [2].

$\vec{\Delta}_{1}$ A position difference distribution in which one node is moving and one is stationary.

W A composite link distance distribution for the Random Waypoint Mobility model.

C A counterexample spatial distribution in which the $G_{R_{v}}$ models provide a poor approximation to $F_{R}$.

Although the subscript may indicate the basic distribution is spatial or a difference distribution, at least approximate link distance distributions are given in each case. For example, $F_{R_{U}}$ is the CDF of the link distance distribution when the underlying spatial distribution is uniform.

Finally, the univariate standard normal CDF is denoted $\Phi(z)$ and its density by $\phi(z)$. The bivariate standard normal CDF is denoted $\Phi_{2}\left(z_{1}, z_{2}\right)$ and its density by $\varphi\left(z_{1}, z_{2}\right)$.

\section{Determining the Link Distance Distribution}

The distribution of the link distance, $R$, may be determined in two different ways. One is to find an exact expression for $F_{R}(r)$ and the other is to obtain an approximation. An exact method produces precise results, but often at a cost of a great effort. The approximate method provides less precise results, but with less effort. The decision of which to use depends on available resources and the need for precision.

\section{II.A. An exact method}

This procedure develops the distribution of $R$ in three steps:

1. From the characteristics of the MANET, first determine $f_{X, Y}(x, y)$, the spatial distribution.

2. From the spatial distribution, determine $f_{\vec{\Delta}}(\vec{\delta})$, the distribution of the coordinate differences.

3. From the difference distribution, then determine $F_{R}(r)$, the distribution of the link distance.

The chief difficulty in this method arises in Step 3. This entails integrating the difference distribution over a circular region. This is often tedious and difficult. Also, the solution tends to differ from model to model.

\section{II.B. An approximate method}

The process below is used for all approximations discussed in this paper. The basic steps are:

1. Determine the standard deviations (and kurtosis if using the $G_{R_{2}}$ estimate) of the marginal spatial distributions of $X$ and $Y$.

2. Verify that the approximation error, $\mathcal{E}_{R}$ is acceptable.

(a) If the error is too large, use the exact method

(b) If the error is acceptable, use either $G_{R_{1}}(r)$ or $G_{R_{2}}(r)$ to approximate $F_{R}(r)$.

The main advantage of this approach is that Step $2 b$ is straightforward. One simply plugs in the appropriate parameter value(s) and cranks out an answer. Also, because the form of $G_{R}(r)$ is the same in each situation, direct comparisons and the construction of composites is easier.

\section{II.C. Approximation details}

This section describes the approximation procedures in detail and illustrates their use by means of an example. It also presents plots of the approximation error for each case.

The original approximation presented in [1] is denoted $G_{R_{0}}$. The approximations presented in this paper are denoted $G_{R_{1}}$ and $G_{R_{2}}$. Each is a modified version of $G_{R_{0}}$. The $G_{R_{0}}$ and $G_{R_{1}}$ approximations differ only in how they determine their parameters. The $G_{R_{2}}$ distribution is a refinement of $G_{R_{1}}$ that also considers the kurtosis of the marginal spatial distributions.

\section{II.C.1. Case 1: equal variances}

If $\sigma_{X}=\sigma_{Y}=\sigma$, then,

$$
G_{R_{1}}(r ; \sigma)=1-\exp \left[-\frac{1}{4}\left(\frac{r}{\sigma}\right)^{2}\right] .
$$


The corresponding estimate from [1] occurs when $h=w$ :

$$
G_{R_{0}}(r ; \kappa, w)=1-\exp \left[-\frac{1}{4}\left(\frac{\kappa r}{w}\right)^{2}\right]
$$

The main difference between these two estimators is that with $G_{R_{0}}$, one needs to determine $\kappa$ for each family of spatial distributions while the $G_{R_{1}}$ estimate proposes that $\kappa=w / \sigma_{X}$. However, to simplify notation, the parameter $\kappa$ is not used. Instead, results are stated directly in terms of $\sigma_{X}$.

If, in addition, the kurtosis of $X$ equals that of $Y$, then

$$
\begin{aligned}
& G_{R_{2}}\left(r: \sigma, \gamma_{2}\right)= \\
& \quad 1-\left\{1-\frac{\gamma_{2}}{256}\left[8\left(\frac{r}{\sigma}\right)^{2}-\left(\frac{r}{\sigma}\right)^{4}\right]\right\} e^{-\frac{1}{4}\left(\frac{r}{\sigma}\right)^{2}},
\end{aligned}
$$

where

$$
\gamma_{2} \triangleq \alpha_{4}-3 \triangleq \frac{E\left[(X-\mu)^{4}\right]}{\sigma^{4}}-3
$$

is the kurtosis ${ }^{1}$ of $X$. Note that when the kurtosis is zero, the $G_{R_{2}}$ and $G_{R_{1}}$ estimates are identical.

\section{II.C.2. Case 2: unequal variances}

If $\sigma_{X} \neq \sigma_{Y}$, then matters are a bit more complicated. The approximate link distance density function is:

$$
g_{R_{1}}(r)=2 \sqrt{a^{2}-b^{2}} I_{0}\left(b r^{2}\right) r e^{-a r^{2}} .
$$

This is based on Eq. (3.6a) in [1], except that:

$$
a=\frac{1}{8}\left(\frac{1}{\sigma_{X}^{2}}+\frac{1}{\sigma_{Y}^{2}}\right), \quad b=\frac{1}{8}\left(\frac{1}{\sigma_{X}^{2}}-\frac{1}{\sigma_{Y}^{2}}\right) .
$$

In Eq. (5), $I_{0}(z)$ is a modified Bessel function of the first kind $[3, \S 9.6 .16]$. Values of $I_{0}(z)$ can be determined directly in most spreadsheets and analytical software ${ }^{2}$. However, Eq. (5) is only the density. To approximate the CDF of $R$, one must integrate the expression

$$
G_{R_{1}}(r)=2 \sqrt{a^{2}-b^{2}} \int_{0}^{r} I_{0}\left(b v^{2}\right) v e^{-a v^{2}} d v .
$$

Even though evaluation of (6) requires numerical methods, it is of the same expression for all underlying spatial distributions. Only the values of $a$ and $b$ will differ. In the exact method, the entire expression may differ from case to case. Thus, the approximation still offers some savings in effort when exploring more than one spatial distribution.

When $h \neq w$, the expressions for $g_{R_{0}}(r)$ and $G_{R_{0}}(r)$ are also given by Eqs. (5) and (6), respectively. However:

$$
\begin{aligned}
a & =\frac{1}{8}\left[\left(\frac{\kappa_{X}}{w}\right)^{2}+\left(\frac{\kappa_{Y}}{h}\right)^{2}\right] \text { and } \\
b & =\frac{1}{8}\left[\left(\frac{\kappa_{X}}{w}\right)^{2}-\left(\frac{\kappa_{Y}}{h}\right)^{2}\right]
\end{aligned}
$$

\footnotetext{
${ }^{1}$ The term "kurtosis" is used in at least two ways in the literature. Some authors refer to $\alpha_{4}$ as the kurtosis and others refer to $\gamma_{2}$, as above. The term $\gamma_{2}$ is used here because it simplifies expressions slightly. Also, because $\gamma_{2}=0$ for a normal random variable, one can see immediately from the magnitude of $\gamma_{2}$ the degree to which the distribution is non-normal.

${ }^{2}$ For example, in Excel, the function is $\operatorname{BESSELI}(z, 0)$.
}

Table 1: Absolute Errors For Example 1

\begin{tabular}{|c|c|c||c|c|}
\hline \multirow{2}{*}{ Approximation } & \multicolumn{3}{|c|}{$\left|F_{R_{U}}(r)-G_{R_{U_{v}}}(r)\right|$} \\
\cline { 2 - 5 } & \multicolumn{2}{|c|}{$h=w$} & \multicolumn{2}{c|}{$h=2 w$} \\
\cline { 2 - 5 } & Max & Mean & Max & Mean \\
\hline \hline$G_{R_{U_{0}}}(r)$ & 0.085 & 0.043 & 0.060 & 0.040 \\
\hline$G_{R_{U_{1}}}(r)$ & 0.054 & 0.022 & 0.044 & 0.020 \\
\hline$G_{R_{U_{2}}}(r)$ & 0.015 & 0.007 & - & - \\
\hline
\end{tabular}

This corresponds to Eq. (3.6d) in [1]. Setting $\kappa_{X}=w / \sigma_{X}$ and $\kappa_{Y}=h / \sigma_{Y}$ in the $G_{R_{0}}$ estimate leads to the results for the $G_{R_{1}}$ estimate.

This paper presents no results for the $G_{R_{2}}(r)$ estimate for cases in which $\sigma_{X} \neq \sigma_{Y}$ or $\gamma_{2_{X}} \neq \gamma_{2_{Y}}$.

Example 1 - Uniform Spatial Distribution: Nodes are uniformly distributed within a $w$ by h rectangle.

Here, $\sigma_{X}=w / \sqrt{12}$, and $\sigma_{Y}=h / \sqrt{12}$. Also, $\gamma_{2_{X}}=$ $\gamma_{2_{Y}}=-1.2$. The difference distribution for $X$ is:

$$
f_{\Delta_{X_{U}}}(\delta)=\frac{w-|\delta|}{w^{2}}, \quad-w \leq \delta \leq w
$$

Case 1, $\mathbf{h}=\mathbf{w}$ : the exact expression for $F_{R_{U}}(r)$ is shown in Part (a) of Figure 1 on Page 4. Substituting $\sigma=w / \sqrt{12}$ into Eq. (1) leads to:

$$
G_{R_{U_{1}}}(r ; w)=1-e^{-3\left(\frac{r}{w}\right)^{2}} .
$$

From Eq. (3), since $\gamma_{2}=-1.2$, we get

$$
G_{R_{U_{2}}}(r)=1-\left\{1+\frac{9}{40}\left[2\left(\frac{r}{w}\right)^{2}-3\left(\frac{r}{w}\right)^{4}\right]\right\} e^{-3\left(\frac{r}{w}\right)^{2}} .
$$

Finally, [1] initially used $\kappa=3$. At that value,

$$
G_{R_{U_{0}}}(r)=1-e^{-\frac{9}{4}\left(\frac{r}{w}\right)^{2}} .
$$

Figure 2 plots the approximation errors for this case. Note that since the ordinate in this plot is the normalized distance $r / w$, it describes the estimation errors in any square region in which nodes are uniformly distributed. The maximum and average of the absolute errors are also listed in Table 1.

Discussion: clearly, the $G_{R_{0}}$ and $G_{R_{1}}$ approximations are computationally much simpler than the exact expression. The maximum absolute error of $G_{R_{U_{0}}}$ is about $60 \%$ greater than that of the $G_{R_{U_{1}}}$ estimate. However, it is small enough to explain the promising results in [1]. The $G_{R_{U_{1}}}$ estimate in turn, has about three and a half times the maximum error of $G_{R_{U_{2}}}$. The average of the absolute error for $G_{R_{U_{0}}}$ is twice that of $G_{R_{U_{1}}}$ and six times that of $G_{R_{U_{2}}}$.

The computations involved in the $G_{R_{U_{1}}}$ estimate are about the same as that of $G_{R_{U_{0}}}$, but the $G_{R_{U_{1}}}$ estimate is superior, due to its lower error. The $G_{R_{U_{2}}}$ estimate is the most precise, at the expense of a few extra calculations and the need to know the kurtoses of the marginal spatial distributions. Also, to use the $G_{R_{U_{2}}}$ estimate, it must be true that $\sigma_{X}=\sigma_{Y}$ and $\gamma_{2_{X}}=\gamma_{2_{Y}}$. 
(a) When $h=w$. Here, $\xi=r / w$.

$$
F_{R}(w \xi \mid w=h)=\left\{\begin{array}{cc}
0, & \xi<0 \\
\xi^{2}\left[\frac{1}{2} \xi^{2}-\frac{8}{3} \xi+\pi\right] & 0 \leq \xi \leq 1 \\
\frac{4}{3}\left(2 \xi^{2}+1\right) \sqrt{\xi^{2}-1}-\left[\frac{1}{2} \xi^{4}+2 \xi^{2}-\frac{1}{3}\right] & \\
+2 \xi^{2}\left[\sin ^{-1}\left(\frac{1}{\xi}\right)-\cos ^{-1}\left(\frac{1}{\xi}\right)\right] & 1 \leq \xi \leq \sqrt{2} \\
1 & \xi>\sqrt{2}
\end{array}\right.
$$

(b) The general case. Here, $\xi=r / w$ and $\zeta=w / h$. Also, $\zeta$ is assumed to be less than one.

$$
F_{R}(w \xi ; \zeta)=\left\{\begin{array}{cc}
0, & \xi<0 \\
\zeta \xi^{2}\left[\frac{1}{2} \zeta \xi^{2}-\frac{4}{3} \xi(1+\zeta)+\pi\right], & 0 \leq \xi \leq 1 \\
\frac{2}{3} \zeta\left(2 \xi^{2}+1\right) \sqrt{\xi^{2}-1}-\frac{1}{6} \zeta\left(8 \xi^{3}+6 \zeta \xi^{2}-\zeta\right)+2 \zeta \xi^{2} \sin ^{-1}(1 / \xi), & 1 \leq \xi \leq \frac{1}{\zeta} \\
\frac{2}{3} \zeta\left(2 \xi^{2}+1\right) \sqrt{\xi^{2}-1}-\frac{1}{2} \zeta^{2}\left[\xi^{4}+2 \xi^{2}-\frac{1}{3}\right] & \\
+\frac{2}{3}\left(2 \zeta^{2} \xi^{2}+1\right) \sqrt{\xi^{2}-\frac{1}{\zeta^{2}}}+\frac{1}{6} \zeta^{-2}-\xi^{2} & \\
+2 \zeta \xi^{2}\left[\sin ^{-1}\left(\frac{1}{\xi}\right)-\cos ^{-1}\left(\frac{1}{\xi}\right)\right], & \frac{1}{\zeta} \leq \xi \leq \sqrt{1+\frac{1}{\zeta^{2}}} \\
1, & \xi>\sqrt{1+\frac{1}{\zeta^{2}}}
\end{array}\right.
$$

Figure 1: Exact Results for the Uniform Cases, Adapted from [1, Eqs. (2.8) and (2.9)].

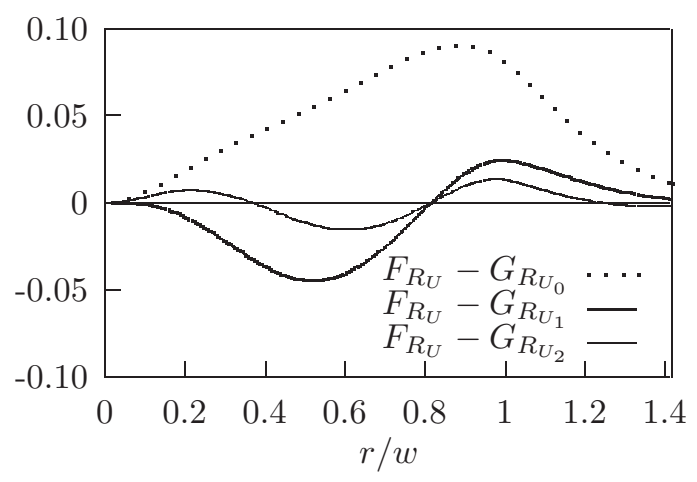

Figure 2: Error Plots for the $G_{R_{n}}$ Estimates When $w=h$ and the Underlying Spatial Distribution is Uniform.

Case 2, $\mathbf{h} \neq \mathbf{w}: \quad$ if $h \neq w$, we can assume, without loss of generality, that $w<h$. The exact result for this case is given by Eq. (b) in Figure 1. Substituting appropriate values of $a$ and $b$ into Eq. (5) yields:

$$
g_{R_{U_{1}}}(r)=\frac{6}{w h} r e^{-a r^{2}} I_{0}\left(b r^{2}\right)
$$

where

$$
a=\frac{3}{2}\left(\frac{1}{w^{2}}+\frac{1}{h^{2}}\right) \text { and } b=\frac{3}{2}\left(\frac{1}{w^{2}}-\frac{1}{h^{2}}\right) .
$$

Figure 3 plots the errors and Table 1 states the maximum and average of the absolute error in this case.

Discussion: in this case, the absolute error of both estimators is slightly less than in the $h=w$ case. Also,

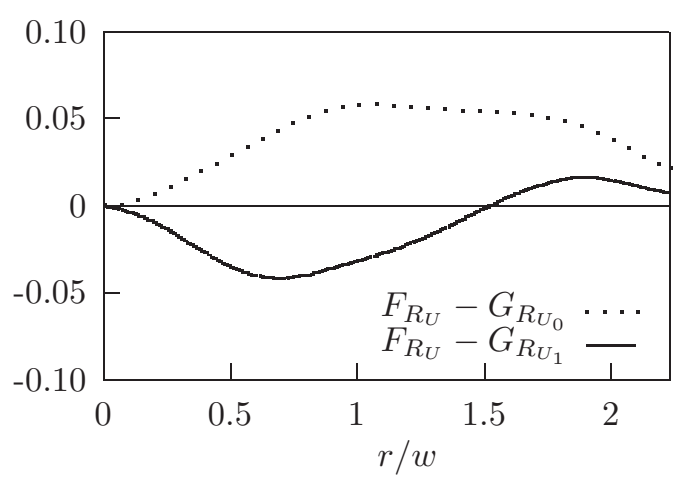

Figure 3: Error Plots for the $G_{R_{n}}$ Estimates when $h=2 w$ and the Underlying Spatial Distribution is Uniform.

the maximum $G_{R_{U_{0}}}$ error is only $35 \%$ greater than that of $G_{R_{U_{1}}}$. The ratio of the average absolute error of $G_{R_{U_{0}}}$ to $G_{R_{U_{1}}}$, however, is still about two. Thus, the $G_{R_{U_{1}}}$ estimate is still superior, though not quite as dominant as in the $h=w$ case.

Note: The following examples focus on the utility of the estimators. In those cases, no exact solution for $F_{R}(r)$ is presented. Instead, the marginal distributions of $\Delta_{X}$ and $\Delta_{Y}$ are stated and exact results are obtained by numerical integration. Also, to compactly illustrate the error for all three estimators, error plots are only given for the case in which $h=w$. As in Example 1, those for other ratios are similar. Finally, specific expressions in terms of $w$ and $h$ were derived in Example 1 to illustrate the relation between the $G_{R_{0}}$ and the other estimates. In practice, this is not nec- 


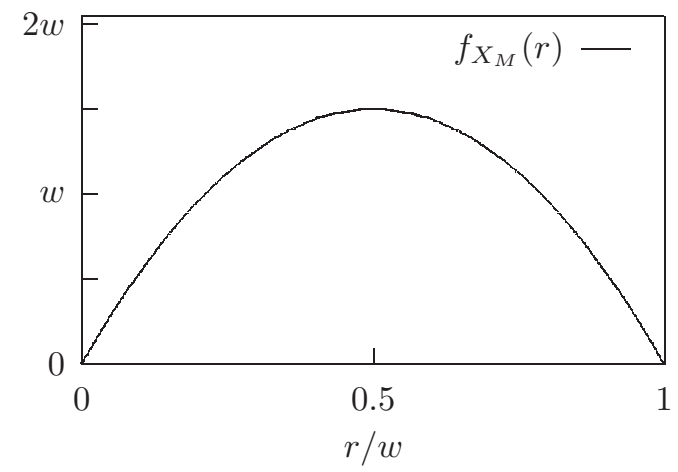

Figure 4: Spatial Distribution for Nodes in Motion [2, Fig. 3.6]

essary. Results are determined in the following examples in the manner in which the estimators would typically be used. That is by substituting parameter values directly into Eqs. (1) or (3), rather than deriving new expressions.

\section{Applications to the Random Waypoint Mobility Model}

There are several versions of the Random Waypoint Mobility Model. The one used here is from [4, p. 160]. In this version, nodes are initially distributed according to a bivariate uniform distribution within a $w$ by $h$ rectangle. After a random dwell time, a node selects a next location, following the same uniform distribution. It then moves in a straight line toward that location at a random speed. Once there, the node once again pauses for a random dwell time and then repeats the process.

In this model, when the nodes are stationary, they are distributed uniformly. However, when they are in motion, they have a different spatial distribution. When a node selects a new location, all points within the $w$ by $h$ rectangle are equally likely to be selected. However, if the node is near an edge, there will be more points in the direction of the interior than not. Thus, the next location is more likely to be in the direction of the interior. As a result, points in the interior of the region are more likely to be on a path than those closer to the boundary.

\section{III.A. A distribution of nodes in motion}

In a recent paper, the authors presented a distribution that looks promising. They derived an exact solution for a onedimensional version of the model, then extrapolated it to the two dimensional case. The authors pointed out that although the two-dimensional version is not an exact solution, it is consistent with simulation results [2]. The marginal density for this distribution is given by Eq. (9) and depicted in Figure 4. It is the "exact" spatial distribution in Example 2 and Section III.B.
Table 2: Absolute Errors For The Waypoint Mobility Model when $h=w$ and $n$ nodes are in Motion

\begin{tabular}{|c|l|l||l|l|}
\hline \multirow{2}{*}{ Approximation } & \multicolumn{3}{|c|}{$\left|F_{R_{W}}(r)-G_{R_{v}}(r)\right|$} \\
\cline { 2 - 5 } & \multicolumn{2}{|c|}{$n=0$} & \multicolumn{2}{c|}{$n=2$} \\
\cline { 2 - 5 } & Max & Mean & Max & Mean \\
\hline \hline$G_{R_{0}}(r ; w, n)$ & 0.085 & 0.043 & 0.275 & 0.137 \\
\hline$G_{R_{1}}(r ; w, n)$ & 0.054 & 0.022 & 0.031 & 0.011 \\
\hline$G_{R_{2}}(r ; w, n)$ & 0.015 & 0.007 & 0.008 & 0.002 \\
\hline
\end{tabular}

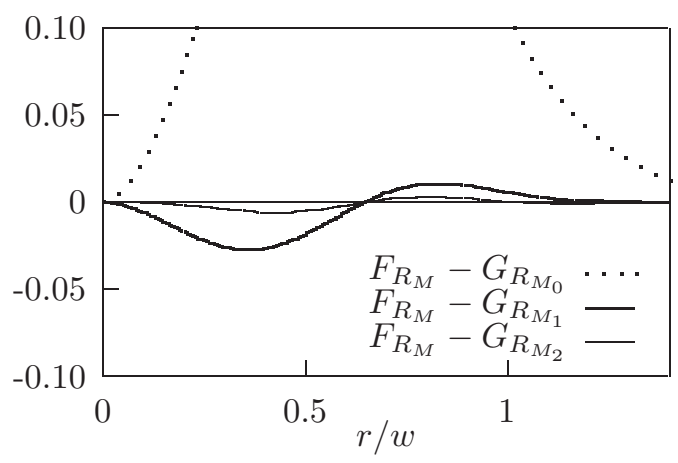

Figure 5: Error Plots for Estimates of $F_{R}(r)$ When the Nodes Are in Constant Motion and $h=w$

Example 2 - Moving Nodes: Nodes are in motion, within a w by hectangle, following a Random Waypoint Mobility Model with zero dwell time.

The marginal density for the spatial distribution is:

$$
f_{X_{M}}(x)=\frac{6 x}{w^{2}}\left(1-\frac{x}{w}\right) .
$$

The standard deviation of $X$ is $w / \sqrt{20}$ and that of $Y$ is $h / \sqrt{20}$. The kurtosis of $X$ and $Y$ is $-6 / 7 \approx-0.86$. From Eq. (31), the marginal difference distribution for $X$ is:

$$
f_{\Delta_{X_{M}}}(\delta)=\frac{6 w^{5}-30 w^{3} \delta^{2}+30 w^{2}\left|\delta^{3}\right|-6\left|\delta^{5}\right|}{5 w^{6}} .
$$

$f_{\Delta_{Y_{M}}}$ is similar with $h$ replacing $w$. Because $X$ and $Y$ are independent, the joint difference density is:

$$
f_{\vec{\Delta}_{M}}\left(\delta_{X}, \delta_{Y}\right)=f_{\Delta_{X_{M}}}\left(\delta_{X}\right) f_{\Delta_{Y_{M}}}\left(\delta_{Y}\right) .
$$

Plots of the approximation errors for $w=h$ are shown in Figure 5. Note that the error for $G_{R_{W_{0}}}$ is off the chart. The maximum and average errors are listed in Table 2.

Discussion: the error for the $G_{R_{0}}$ approximation with $\kappa=3$ is immense, while that of the $G_{R_{1}}$ and $G_{R_{2}}$ estimates is actually less than in Example 1. The $G_{R_{1}}$ and $G_{R_{2}}$ functions automatically adapt to differences in the underlying distributions. Thus, one may easily include the impact of those differences in analysis. The next section illustrates this point. 
Table 3: Parameters for $\Delta_{X_{n}}$ in the Waypoint Model

\begin{tabular}{|c|c|c|c|c|}
\hline r.v. & $p_{n}$ & $\sigma_{\Delta_{X_{n}}}^{2}$ & $\gamma_{\Delta_{X_{n}}}$ & $E\left[\Delta_{X}^{4}\right]$ \\
\hline \hline$\Delta_{X_{0}}$ & $(1-p)^{2}$ & $w^{2} / 6$ & $-3 / 5$ & $w^{4} / 15$ \\
\hline$\Delta_{X_{1}}$ & $2 p(1-p)$ & $2 w^{2} / 15$ & $-33 / 56$ & $3 w^{4} / 70$ \\
\hline$\Delta_{X_{2}}$ & $p^{2}$ & $w^{2} / 10$ & $-3 / 7$ & $9 w^{4} / 350$ \\
\hline
\end{tabular}

\section{III.B. A general Waypoint distribution}

Consider the following situation.

Example 3 - A Waypoint Model: Nodes within a $w$ by $h$ rectangle, travel according to the Random Waypoint Mobility Model. The probability that a randomly-selected node is in motion, given that it is communicating, is $p$.

Because nodes in motion have a different spatial distribution than those that are stationary, there are three different difference distributions:

- That when both nodes are in motion,

- That when both nodes are stationary, and

- That when one node is in motion and one is stationary.

Let $\vec{\Delta}_{n}$ be the random variable representing the geographical difference for a communications link in which $n=$ $0,1,2$ nodes of the pair are in motion. The marginal distribution of $\Delta_{X_{0}}$ is given by Eq. (7) and that for $\Delta_{X_{2}}$ by Eq. (10). For $\Delta_{X_{1}}$, the difference distribution can be derived from Eq. (31) using the uniform density for one $f_{X}$ term and Eq. (9) for the other. The result is:

$$
\begin{aligned}
f_{\Delta_{X_{1}}}(\delta) & =\frac{1}{2} \int_{|\delta|}^{2 w-|\delta|}\left[\frac{1}{w}\right]\left[\frac{3(s-\delta)(2 w-s+\delta)}{2 w^{3}}\right] d s \\
& =\frac{w^{3}-3 w \delta^{2}+2\left|\delta^{3}\right|}{w^{4}}
\end{aligned}
$$

Note that $X_{1}$ and $X_{2}$ are not i.i.d., since one being in motion means the other is not. However, either may be the one in motion, so this distribution is still symmetric. For this distribution, $\sigma_{\Delta_{X_{1}}}=w \sqrt{2 / 15}$ and $\gamma_{2_{\Delta_{X_{1}}}}=-33 / 56 \approx$ -0.59 . Although these parameters are for $\Delta_{X_{1}}$ and not $X$, this is no serious problem. From Eq. (32),

$$
\sigma_{X}=\sigma_{\Delta_{X}} / \sqrt{2}
$$

Also, from Eq. (36),

$$
\gamma_{2_{X}}=2 \gamma_{2_{\Delta_{X}}}
$$

Hence, we can use the $G_{R_{1}}$ and $G_{R_{2}}$ estimates with $\sigma=$ $w / \sqrt{15}$ and $\gamma_{2}=-33 / 28 \approx-1.18$.

Let $\Delta_{X_{W}}$ represent the composite marginal geographical difference considering all three possibilities above. If $\sigma_{X_{n}}$ is the standard deviation of $\Delta_{X_{n}}$ and $p_{n}$ is the probability that a link has $n$ nodes in motion, then the variance of the composite marginal distribution is:

$$
\sigma_{\Delta_{X_{W}}}^{2}=p_{0} \sigma_{\Delta_{0}}^{2}+p_{1} \sigma_{\Delta_{1}}^{2}+p_{2} \sigma_{\Delta_{2}}^{2}
$$

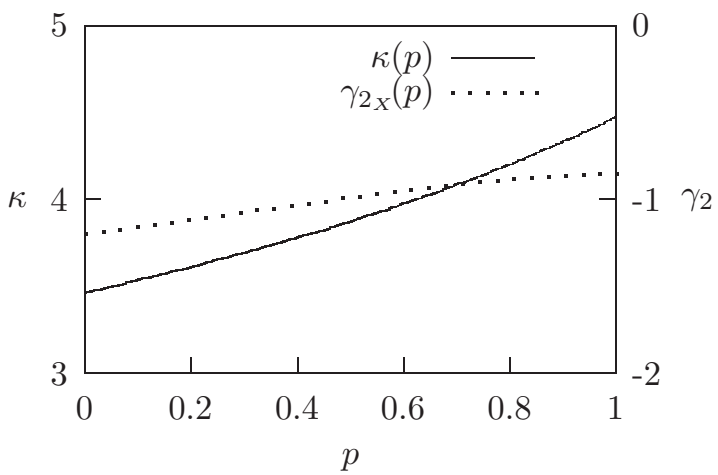

Figure 6: Plots of $\kappa$ and $\gamma_{2_{X}}$ as a Function of $p$ in the Random Waypoint Mobility Model

Table 3 lists the probability of 1, 2, or 3 nodes of a communicating pair being in motion. It also lists the variance of each difference distribution. Using these values, the variance of the composite is:

$$
\begin{aligned}
\sigma_{\Delta_{X_{M}}}^{2} & =w^{2}\left[\frac{(1-p)^{2}}{6}+\frac{4 p(1-p)}{15}+\frac{p^{2}}{10}\right] \\
& =w^{2}\left(\frac{5-2 p}{30}\right) .
\end{aligned}
$$

Using Eq. (13) leads to:

$$
\sigma_{X_{W}}=w \sqrt{\frac{5-2 p}{60}} .
$$

Using a similar argument and other information in Table 3, the fourth moment of $\Delta_{X_{W}}$ is:

$$
\begin{aligned}
E & {\left[\Delta_{X_{W}}^{4}\right] } \\
& =(1-p)^{2} E\left[\Delta_{X_{0}}^{4}\right]+2 p(1-p) E\left[\Delta_{X_{1}}^{4}\right]+p^{2} E\left[\Delta_{X_{2}}^{4}\right] \\
& =w^{4}\left(\frac{70-50 p+7 p^{2}}{1050}\right) .
\end{aligned}
$$

From this, the kurtosis of $\Delta_{X_{W}}$ is:

$$
\begin{aligned}
\gamma_{2_{\Delta_{W}}} & =\frac{E\left[\Delta_{X_{W}}^{4}\right]}{\sigma_{X_{W}}^{4}}-3 \\
& =\frac{-105+120 p-42 p^{2}}{7(5-2 p)^{2}} .
\end{aligned}
$$

Using Eq. (14) leads to:

$$
\gamma_{2_{X_{W}}}=\frac{-210+240 p-84 p^{2}}{7(5-2 p)^{2}} .
$$

Figure 6 shows plots of $\kappa=w / \sigma_{X}$. and $\gamma_{2}$ for all values of $p$. The value of $\kappa$ runs from about 3.5 to 4.5 , which explains the poor performance of $G_{R_{0}}$ at $\kappa=3$. Although $\gamma_{2}$ does not vary much, it is about -1 . This explains why the $G_{R_{2}}$ estimate is significantly more accurate than the others.

Figure 7 shows plots of $G_{R_{W_{2}}}(r ; p)$ for a square region. Figure 8 shows plots of $G_{R_{W_{1}}}(r ; p)$ for a rectangular region. Both plots are for $p=0$ through $p=1$ in steps of 


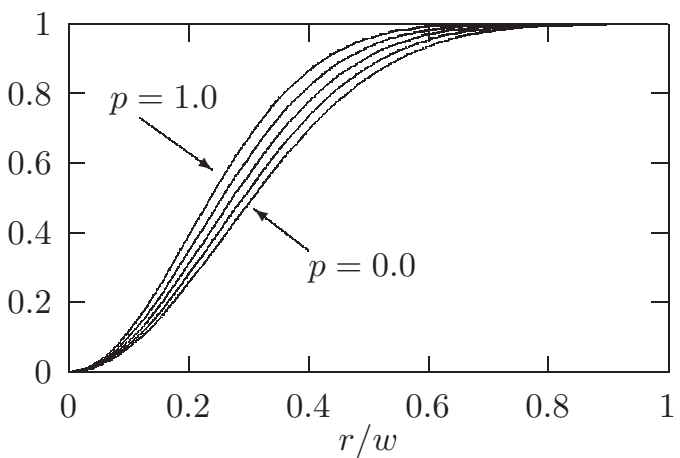

Figure 7: Plots of $G_{R_{2}}(r ; p)$ for the Waypoint Distribution for $p=0.00,0.25,0.50,0.75$, and 1.00 when $h=w$

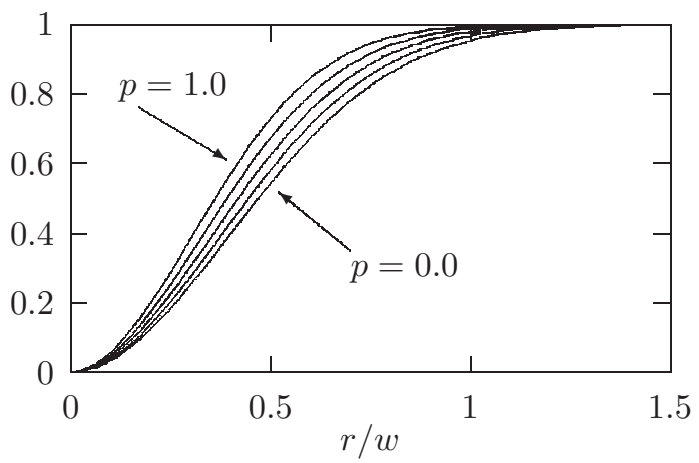

Figure 8: Plots of $G_{R_{1}}(r ; p)$ for the Waypoint Distribution for $p=0.00,0.25,0.50,0.75$, and 1.00 when $h=2 w$

0.25 . Note that when $p=0$, we have the uniform distribution and when $p=1$, we have the distribution of nodes in constant motion. Although approximate, these plots give a good idea of how the probability that a communicating node is in motion affects the distribution of link distances.

The analysis in the exact case would be far more involved but would yield a result that differs from the approximation by only a few probability points. Also, this example illustrates a primary advantage of the $G_{R_{1}}$ and $G_{R_{2}}$ approximations over the $G_{R_{0}}$. The $G_{R_{0}}$ approximation would either ignore the impact of $p$ entirely or require a separate analysis step to determine $\kappa$. The $G_{R_{1}}$ and $G_{R_{2}}$ approximations automatically respond to changes in the distributions and strike a different balance of effort and precision.

\section{Approximation Error}

In the earlier examples, the $G_{R_{1}}$ and $G_{R_{2}}$ estimators had a small to moderate error. However, as shown in the next example, this error can be large.

Example 4 - Counterexample: Nodes are distributed in a square region according to a distribution with the marginal distribution is given by Eq. (21).

The density, also depicted in Figure 9, is:

$$
f_{X_{C}}(x)=\frac{3(2 x-w)^{2}}{w^{3}} .
$$

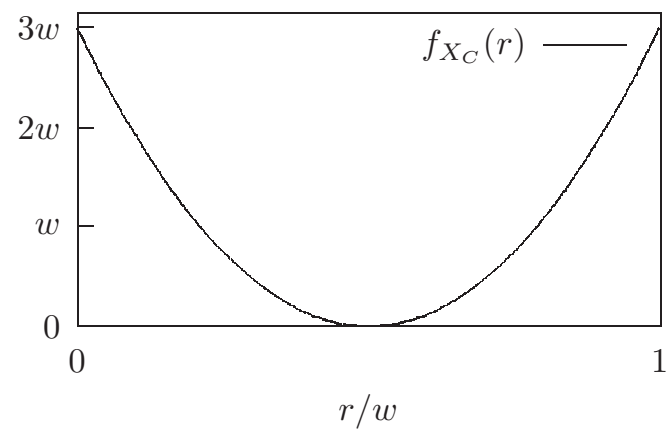

Figure 9: Counterexample's Marginal Spatial Distribution

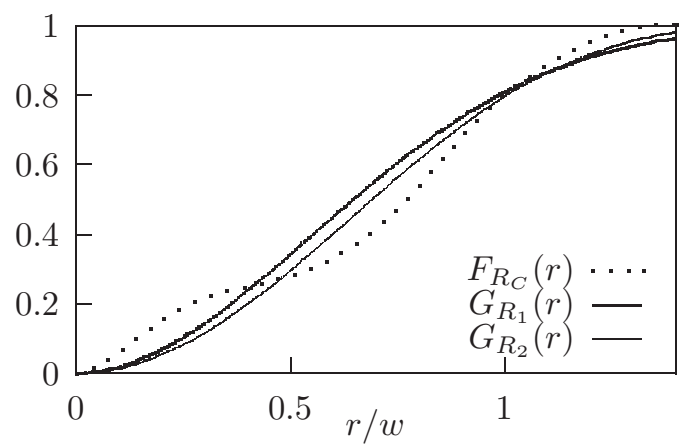

Figure 10: Plots of the Exact and Approximate CDF Functions for $R$ in the Counterexample When " $\mathrm{h}=\mathrm{w}$ "

In this case, nodes would tend to stay near the perimeter of the rectangle. Such a distribution could arise if there were a large geographical feature, such as a mountain or lake in the middle of the region. It could also occur if operational constraints would tend to keep nodes near the perimeter, such as in fighting a forest fire.

Here, $\sigma_{X}=w \sqrt{3 / 20}$ and $\gamma_{2}=-38 / 21 \approx-1.8$. The difference distribution is:

$$
f_{\Delta_{C}}(\delta)=\frac{9 w^{5}-45 w^{4}|\delta|+60 w^{3} \delta^{2}-24\left|\delta^{5}\right|}{5 w^{6}} .
$$

As shown in Table 4, the error is about three times as large as in the uniform case. Rather than showing error plots, Figure 10 shows $F_{R_{C}}$ and its approximations. Note that the approximations are seldom close to the true value.

This rest of this section briefly explains the principles underlying these estimators. It then gives some guidance in detecting large errors.

\section{IV.A. How the estimators work}

In the exact method, one first determines $f_{\vec{\Delta}}$ from $f_{X, Y}$, then derives $F_{R}$ from $f_{\vec{\Delta}}$. The approximations operate on two basic assumptions:

1. The approximate difference distribution, $g_{\vec{\Delta}}$, closely resembles $f_{\vec{\Delta}}$, and

2. The probability that a node lies outside the circle $r=$ $\min (w, h)$ is very small.

As long as these assumptions are valid, the approximations work well. Because $X$ and $Y$ are assumed to be independent, we can examine this issue by looking at the marginal 


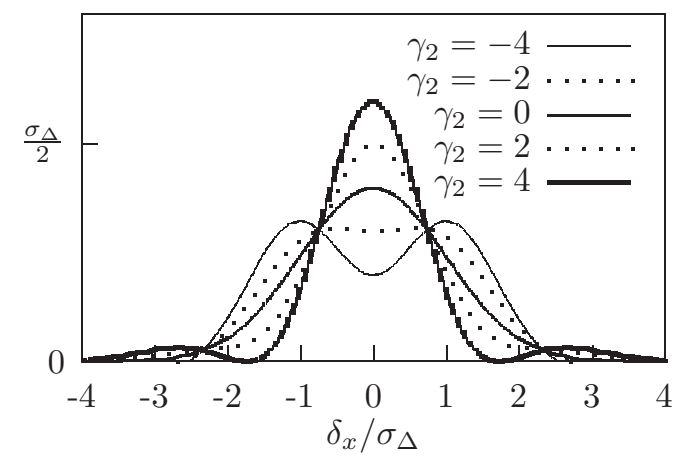

Figure 11: Plots of $g_{\Delta_{X_{2}}}$, the Marginal Difference Density used in the $G_{R_{2}}$ Estimate, for Selected Values of the Kurtosis

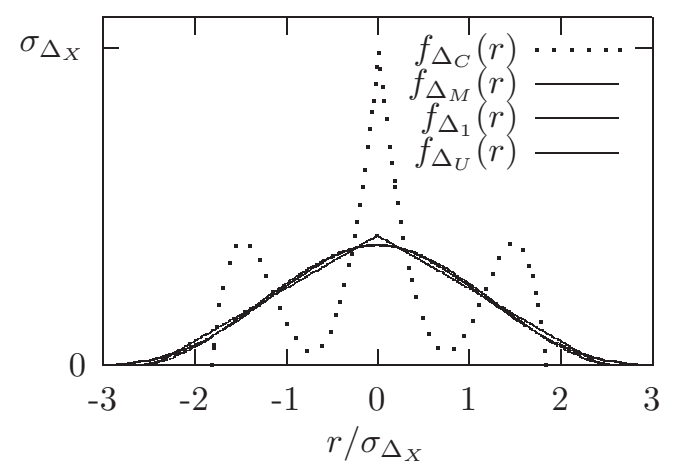

Figure 12: Densities of $\Delta_{X}$ for the Examples

distributions. The following focuses on $\Delta_{X}$, but the arguments also apply to $\Delta_{Y}$.

The $G_{R_{1}}$ estimate employs a bivariate normal approximation to $f_{\vec{\Delta}}$. From Appendix A, the marginal difference distribution shares the following features with a $N\left(0, \sigma_{\Delta_{X}}\right)$ distribution:

- $f_{\Delta_{X}}(\delta)=f_{\Delta_{X}}(-\delta)$ for $-w \leq \delta \leq w$,

- $E\left[\Delta_{X}^{n}\right]=0$ if $n$ is odd, and

- by design, the variances are equal.

Furthermore, The kurtosis of $\Delta_{X}$ is half that of $X$, indicating a closer fit to the normal. Thus, as long as $E\left[\Delta_{X}^{n}\right]$, for some even $n \geq 4$, is not very different from that of a $N\left(0, \sigma_{\Delta}\right)$ r.v., Assumption 1 is likely to be valid.

In the case of $G_{R_{2}}$, the standard deviation and the kurtosis of $g_{\Delta_{X_{2}}}$ match those of $\Delta_{X}$, leading to a closer fit. Figure 11 illustrates the flexibility of $g_{\Delta_{X_{2}}}$. As long as $E\left[\Delta_{X}^{n}\right]$ for some even $n \geq 6$ is not very different from the normal, Assumption 1 is likely to be valid.

The second assumption depends on the fact that link lengths greater than $\min (w, h)$ are rare. For example, in Figure 7, the probability of $r \leq w$ is virtually one for all values of $p$ and in Figure 8, this probability is very close to one, in spite of the fact that $r$ can be as large as $2.24 \mathrm{w}$.

\section{IV.B. Errors in the examples}

In the $R_{W}$ approximation, the assumptions are satisfied, at least to some extent, for all three components $\left(\Delta_{U}, \Delta_{M}\right.$
Table 4: Errors for the Examples in which $h=w$

\begin{tabular}{|c|c|c|c|c|c|c|}
\hline \multirow[b]{2}{*}{ Case } & \multicolumn{2}{|c|}{$\left|F_{R}-G_{R_{1}}\right|$} & \multirow{2}{*}{$\begin{array}{c}G_{R_{1}} \\
p_{b}^{\star}\end{array}$} & \multicolumn{2}{|c|}{$\left|F_{R}-G_{R_{2}}\right|$} & \multirow{2}{*}{$\begin{array}{c}G_{R_{2}} \\
p_{b}^{\star}\end{array}$} \\
\hline & Max & Mean & & Max & Mean & \\
\hline$U$ & 0.054 & 0.022 & 0.050 & 0.015 & 0.007 & 0.039 \\
\hline$M$ & 0.031 & 0.011 & 0.007 & 0.008 & 0.002 & 0.001 \\
\hline$C$ & 0.160 & 0.063 & 0.189 & 0.120 & 0.055 & 0.201 \\
\hline
\end{tabular}

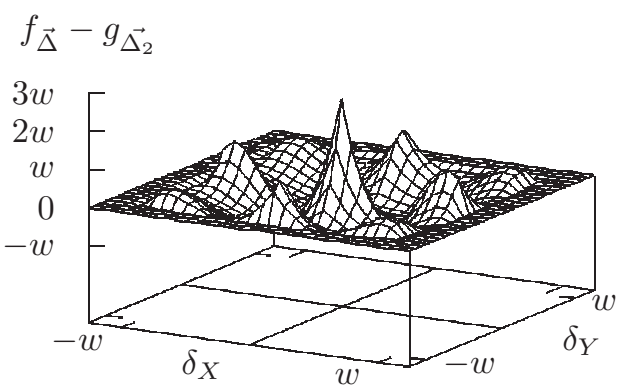

Figure 13: The $G_{R_{2}}$ Error in Estimating the Difference Distribution for the Counter Example

and $\left.\Delta_{1}\right)$. Note that the error,

$$
\begin{aligned}
\mathcal{E}_{W_{v}}(r) \triangleq & F_{R_{W}}(r)-G_{R_{v}}(r) \\
= & (1-p)^{2} \mathcal{E}_{U_{v}}(r)+2 p(1-p) \mathcal{E}_{\Delta_{1_{v}}}(r) \\
& +p^{2} \mathcal{E}_{M_{v}}(r),
\end{aligned}
$$

is a convex combination of the components' errors. Also, $\gamma_{2_{X}}$ monotonically improves with increasing $p$. Thus, we can expect the error for $G_{R_{1}}$ and $G_{R_{2}}$ to be within the limits in Table 2.

On the other hand, both of the assumptions are violated in Example 4. Figure 12 shows the marginal difference densities for the four examples. Definitely, $f_{\Delta_{X_{C}}}$ is very unusual. Also, comparing $f_{\Delta_{X_{C}}}$ to the plots in Figure 11, it is clear that $\Delta_{X_{C}}$ cannot be closely matched by $g_{\Delta_{X_{2}}}$ As for the second assumption, $p_{b}=\operatorname{Pr}\{R>w\}$, when $h=w$, is 0.18 . This probability would be even larger when $h \neq w$. This is significantly higher than $p_{b}$ in the other examples (See Table 4).

It is tempting to suspect that the unusual value of $\gamma_{2_{X}}$ is the basis for this high error. However, the kurtosis of $g_{\Delta_{X_{2}}}$ is identical. Figure 13 shows the error estimating $f_{\vec{\Delta}_{C}}$ with $g_{\vec{\Delta}_{2}}$. This error is about an order of magnitude greater than in the uniform case. Looking at this error plot, it is clear that one needs a higher order approximation to deal with the excursions of $f_{\vec{\Delta}_{C}}$.

\section{IV.C. Identifying high-error situations}

Given that a large error can occur, the question how to detect it. The surest way to determine the approximation error is to compare the exact and approximate distributions of $R$. As shown in Examples 2 through 4, it is not necessary to obtain an explicit expression for $F_{R}(r)$. One may estimate the error by means of numerical integration. While this method reduces the advantage of using the approximations, it does certify the utility of the simpler expressions 
and still avoids the need to find an exact expression for $F_{R}(r)$. Also, it may be the only option in some cases. For example, this method may be used with regions of arbitrary shape or when $X$ and $Y$ are statistically dependent.

The tests below indicate when error is likely to be large, but do not assure it is small. The comparison above should be used if any of the simpler tests fail, or if one needs to be absolutely sure the error is small.

Checking for boundary errors: a simple check is to find

$$
p_{b}^{\star}=1-G_{R_{v}}[\min (x, y)] \approx p_{b} .
$$

This is the probability that at least part of a circle of radius $r$ will fall outside the rectangle. If this value is large, then either $F_{R}[\min (x, y)]$ is significantly less than one or $G_{R_{v}}$ underestimates $F_{R}$ near the boundary. Either way, the truncation error is likely to be large. Table 4 lists values of $p_{b}^{\star}$ for three examples.

Comparing marginals: another indicator of the error is the difference $F_{\left|\Delta_{X}\right|}(r)-G_{\left|\Delta_{X}\right|}(r)$ where $0 \leq r \leq$ $\min (w, h)$,

$$
F_{\left|\Delta_{X}\right|}(r)=2 \int_{0}^{r} f_{\Delta_{X}}(\delta) d \delta
$$

and

$$
G_{\left|\Delta_{X}\right|}(r)=\Phi\left(\frac{r}{\sigma_{X} \sqrt{2}}\right)-\Phi\left(\frac{-r}{\sigma_{X} \sqrt{2}}\right) .
$$

In this comparison, the marginal $\mathrm{CDF}$ provides some insight into how well the marginals of $\vec{\Delta}$ are estimated by those of $g_{\vec{\Delta}}$ within the rectangle. This check would be repeated on the $y$ axis, if the distribution of $Y$ differed. As shown in Figure 14, this computation yields similar maximum absolute errors to the comparison of $G_{R_{1}}$ with $F_{R}$. The functions for the examples are:

$$
\begin{aligned}
F_{|\Delta|_{U}}(r) & =\frac{2 w r-r^{2}}{w^{2}}, \\
F_{|\Delta|_{M}}(r) & =\frac{12 w^{5} r-20 w^{3} r^{3}+15 w^{2} r^{4}-2 r^{6}}{5 w^{6}}, \\
F_{|\Delta|_{C}}(r) & =\frac{18 w^{5} r-45 w^{4} r^{2}+40 w^{3} r^{3}-8 r^{6}}{5 w^{6}} .
\end{aligned}
$$

\section{Conclusion}

This paper explains why the difference distribution is approximately Gaussian, validating the approximate distribution given in [1]. It also presents two estimators of the link distance distribution in a rectangular region that are more robust and more accurate than the one presented in [1].

The paper also verifies that these estimators work well in one version of the Random Waypoint Mobility Model. It also presents a approximation to the link distance distribution in that model that is a function of the probability that a node is in motion.

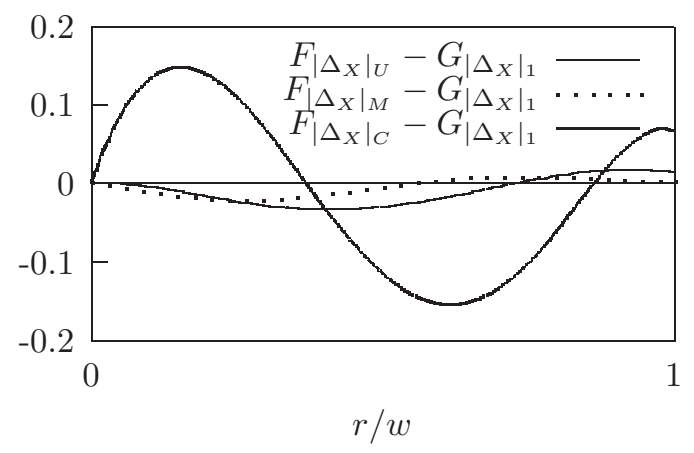

Figure 14: Comparisons of the Marginal Cumulative Functions in Examples 1 Through 3. Note Different Vertical Scale

These would relieve some computational load and provide a theoretical framework for the study of MANETs at this level. Although results are stated in terms of the distribution of $X$, they can be converted to results in terms of $\Delta_{X}$ by means of Eqs. (32) and (36).

The counterexample shows that although the approximations are robust, they have their limits. It also shows that one cannot always characterize a distribution sufficiently on the basis of its first four moments. In the counterexample, $\alpha_{8} \approx 1.2$, and $\alpha_{10} \approx 30$. The same normalized moments for the normal are 105 and 945, respectively.

There are three problems with developing an estimate using higher moments. First of all, there is the technical problem of deriving the expressions for $G_{R_{v}}(r)$. The second problem is that in many practical situations, estimates of these higher moments are likely to be unreliable. Finally, no matter how many moments are included, there will always be the chance that a situation will arise that needs even higher order moments.

This paper demonstrates how to approximate the distribution of link distances in one version of the Random Waypoint Mobility Model. A productive area of further research might be to apply this technique to other mobility models, such as in [5]. In addition, although the $G_{R_{2}}$ estimate seems to be accurate, at this time there is no closed form expression for cases in which $\sigma_{X} \neq \sigma_{Y}$. Finally, a logical next step is to determine distributions for hopcounts, number of neighbors, etc., based on the approximations.

\section{References}

[1] Leonard E. Miller. Distribution of link distances in a wireless network. Journal of Research of the National Institute of Standards and Technology, 106(2):401412, 2001.

[2] Christian Bettstetter and Christian Wagner. The spatial node distribution of the random waypoint mobility model. In the 1st German Workshop on Mobile Ad-Hoc Networks (WMAN'02), Ulm, Germany, 2002. Technische Universität München, Institute of Communication Networks, Munich, Germany. 
[3] Milton Abramowitz and Irene A. Stegun. Handbook of Mathematical Functions. Dover, New York, NY, 1965. (December 1972 printing.).

[4] David B. Johnson, David A. Maltz, and A Josh Broch. DSR: The Dynamic Source Routing Protocol for MultiHop Wireless Ad Hoc Networks, chapter 5, pages 139 - 172. Addison-Wesley, New York, NY, 2001.

[5] T. Camp, J. Boleng, and V. Davies. A survey of mobility models for ad hoc network research. Wireless Communications \& Mobile Computing (WCMC): Special issue on Mobile Ad Hoc Networking: Research, Trends and Applications, 2(5):483-502, 2002.

[6] Erwin Kreyszig. Advanced Engineering Mathematics. Wiley, New York, NY, seventh edition, 1993.

[7] Norman L. Johnson and Samuel Kotz. Continuous Univariate Distributions, volume I. John Wiley \& Sons, New York, NY, 1970.

[8] S. Blinnikov and R. Moessner. Expansions for nearly gaussian distributions. Astronomy \& Astrophysics, Supplement Series, 130:193 - 205, May 1998.

\section{A. The Distribution of the Geograph- ical Difference}

This section develops the distribution of the random variable $\Delta_{X}=X_{1}-X_{2}$ as well as its key properties. This development assumes that the $X_{i}$ are continuous random variables on $[0, w]$ with a common continuous density, $f_{X}(x)$, that is differentiable on $(0, w)$. It also assumes that $X_{1}$ and $X_{2}$ are statistically independent. The discussion is in terms of $X$, but also applies to $Y$, with $h$ replacing $w$.

\section{A.A. Deriving the distribution of $\Delta_{\mathrm{X}}$}

This distribution may be derived from that of $X$ using the Change of Variable Technique [6, p. 519], with $\Sigma_{X}=$ $X_{1}+X_{2}$ and $\Delta_{X}=X_{1}-X_{2}$. The inverse functions are $x_{1}(s, \delta)=(s+\delta) / 2$ and $x_{2}(s, \delta)=(s-\delta) / 2$. The Jacobian is

$$
J=\left|\begin{array}{cc}
\frac{\partial x_{1}}{\partial s} & \frac{\partial x_{1}}{\partial \delta} \\
\frac{\partial x_{2}}{\partial s} & \frac{\partial x_{2}}{\partial \delta}
\end{array}\right|=\left|\begin{array}{rr}
\frac{1}{2} & \frac{1}{2} \\
\frac{1}{2} & -\frac{1}{2}
\end{array}\right|=-\frac{1}{2} .
$$

Since the Jacobian is of one sign for all permitted values of $(s, \delta)$, the joint density of $\Sigma_{X}$ and $\Delta_{X}$ is:

$$
\begin{aligned}
f_{\Sigma_{X}, \Delta_{X}}(s, \delta) & =|J| f_{X_{1}}\left(\frac{s+\delta}{2}\right) f_{X_{2}}\left(\frac{s-\delta}{2}\right) \\
& =\frac{1}{2} f_{X}\left(\frac{s+\delta}{2}\right) f_{X}\left(\frac{s-\delta}{2}\right) .
\end{aligned}
$$

From Eq. (30) we can find $f_{\Delta}(\delta)$ by integrating over all permitted values of $s$. The result is:

$$
f_{\Delta_{X}}(\delta)=\frac{1}{2} \int_{|\delta|}^{2 w-|\delta|} f_{X}\left(\frac{s+\delta}{2}\right) f_{X}\left(\frac{s-\delta}{2}\right) d s .
$$

\section{A.B. Difference distribution properties}

Because $\Delta_{X}$ is the difference of two i.i.d. random variables its variance is the sum of their variances. Hence,

$$
\sigma_{\Delta_{X}}=\sigma_{X} \sqrt{2}
$$

From Eq. (31), it is clear that $f_{\Delta_{X}}(\delta)=f_{\Delta_{X}}(-\delta)$. Since $\Delta_{X}$ is defined on the interval $[-w, w]$, the following is true of its $r$-th central moment,

$$
\begin{aligned}
E\left[\Delta_{X}^{r}\right] & =\int_{-w}^{0} \delta^{r} f_{\Delta_{X}}(\delta) d \delta+\int_{0}^{w} \delta^{r} f_{\Delta_{X}}(\delta) d \delta \\
& =\left\{\begin{array}{cc}
2 \int_{0}^{w} \delta^{r} f_{\Delta_{X}}(\delta) d \delta, & \text { if } r \text { is even } \\
0 & \text { if } r \text { is odd }
\end{array}\right.
\end{aligned}
$$

Thus, $E\left[\Delta_{X}\right]=0$ and $\sigma_{\Delta_{X}}^{2}=E\left[\Delta_{X}^{2}\right]$. Also, all odd moments of $\Delta_{X}$ are equal to zero. In addition, $F_{\Delta_{X}}(0)=0.5$.

In addition, the kurtosis of $\Delta_{X}$ and that of the underlying $X$ distribution are related. First of all,

$$
\begin{aligned}
\alpha_{4}\left(\Delta_{X}\right) & \stackrel{\Delta}{=}\left[\left(\Delta_{X}-E\left[\Delta_{X}\right]\right)^{4}\right]=E\left[\left(X_{1}-X_{2}\right)^{4}\right] \\
& =2 E\left[X^{4}\right]-8 E\left[X^{3}\right] \mu_{X}+6 E^{2}\left[X^{2}\right] .
\end{aligned}
$$

The last line is the result of the $X_{i}$ being i.i.d. Looking at the last term and using that fact that $\sigma_{X}^{2}=E\left[X^{2}\right]-\mu_{X}^{2}$ twice leads to:

$$
\begin{aligned}
E^{2}\left[X^{2}\right] & =\left(\sigma_{X}^{2}+\mu_{X}^{2}\right)^{2} \\
& =\sigma_{X}^{4}+2 \sigma_{X}^{2} \mu_{X}^{2}+\mu_{X}^{4} \\
& =\sigma_{X}^{4}+2\left(E\left[X^{2}\right]-\mu_{X}^{2}\right) \mu_{X}^{+} \mu_{X}^{4} \\
& =\sigma_{X}^{4}+2 E\left[X^{2}\right] \mu_{X}^{2}-\mu_{X}^{4} .
\end{aligned}
$$

Thus, we have from Eqs. (4), (32) and (35),

$$
\begin{aligned}
\gamma_{2_{\Delta_{X}}} \triangleq & \frac{\alpha_{4}\left(\Delta_{X}\right)}{\sigma_{\Delta_{X}}^{4}}-3=\frac{\alpha_{4}\left(\Delta_{X}\right)-12 \sigma_{X}^{4}}{4 \sigma_{X}^{4}} \\
= & \frac{2 E\left[X^{4}\right]-8 E\left[X^{3}\right] \mu_{X}-6 \sigma_{X}^{4}}{4 \sigma_{X}^{4}} \\
& +\frac{12 E\left[X^{2}\right] \mu_{X}^{2}-6 \mu_{X}^{4}}{4 \sigma_{X}^{4}} \\
= & \frac{1}{2}\left[\frac{E\left[\left(X-\mu_{X}\right)^{4}\right]}{\sigma_{X}^{4}}-3\right] \\
= & \frac{1}{2} \gamma_{2_{X}} .
\end{aligned}
$$

\section{B. Development of the $\mathrm{G}_{\mathrm{R}_{1}}$ Approxi- mation}

The development of $G_{R_{1}}$ is very similar to that of $G_{R_{0}}$ in [1, pp. $407 \mathrm{ff}]$. However, this development starts with the results of Appendix A, and does not employ the parameter $\kappa$. It also is based on the distribution of $X$, rather than that of $\Delta_{X}$.

Assume $\vec{\Delta}=\left(\delta_{X}, \delta_{Y}\right)$ is a bivariate normal random variable with $\vec{\mu}=(0,0)$ and $\vec{\sigma}=\left(\sigma_{\Delta_{X}}, \sigma_{\Delta_{Y}}\right)$. Assuming that $\Delta_{X}$ and $\Delta_{Y}$ are uncorrelated, the density of $\vec{\Delta}$ is:

$$
g_{\vec{\Delta}}\left(\delta_{X}, \delta_{Y}\right)=\frac{1}{2 \pi \sigma_{\Delta_{X}} \sigma_{\Delta_{Y}}} e^{-\frac{1}{2}\left[\left(\frac{\delta_{X}}{\sigma_{\Delta}}\right)^{2}+\left(\frac{\delta_{Y}}{\sigma_{\Delta_{Y}}}\right)^{2}\right]}
$$




$$
=\frac{1}{4 \pi \sigma_{X} \sigma_{Y}} e^{-\frac{1}{4}\left[\left(\frac{\delta_{X}}{\sigma_{X}}\right)^{2}+\left(\frac{\delta_{Y}}{\sigma_{Y}}\right)^{2}\right]} .
$$

The Jacobian for the change of variable $\delta_{X}=r \cos \theta$ and $\delta_{Y}=r \sin \theta$ is $r$, so we can rewrite Eq. (37) as:

$$
g_{\vec{\Delta}}(r, \theta)=\frac{r}{4 \pi \sigma_{X} \sigma_{Y}} e^{-\frac{r^{2}}{4}\left[\frac{\cos ^{2} \theta}{\sigma_{X}^{2}}+\frac{\sin ^{2} \theta}{\sigma_{Y}^{2}}\right]} .
$$

To find the marginal distribution of $R$, integrate (38) over a circle of radius $r$.

$$
\begin{aligned}
g_{R}(r) & =\frac{r}{4 \pi \sigma_{X} \sigma_{Y}} \int_{0}^{2 \pi} e^{-\frac{r^{2}}{4}\left[\frac{\cos ^{2} \theta}{\sigma_{X}^{2}}+\frac{\sin ^{2} \theta}{\sigma_{Y}^{2}}\right]} d \theta \\
& =\frac{r}{\pi \sigma_{X} \sigma_{Y}} \int_{0}^{\frac{\pi}{2}} e^{-\frac{r^{2}}{4}\left[\frac{\cos ^{2} \theta}{\sigma_{X}^{2}}+\frac{\sin ^{2} \theta}{\sigma_{Y}^{2}}\right]} d \theta .
\end{aligned}
$$

The last step, which follows from the symmetry in each quadrant of the circle, simplifies evaluation, since $\sin \theta$ and $\cos \theta$ are non-negative in the first quadrant.

If $\sigma_{\mathbf{X}}=\sigma_{\mathbf{Y}}=\sigma:$ then,

$$
g_{R}(r)=\frac{r}{\pi \sigma^{2}} e^{-\frac{1}{4}\left(\frac{r}{\sigma}\right)^{2}} \int_{0}^{\frac{\pi}{2}} d \theta=\frac{r}{2 \sigma^{2}} e^{-\frac{1}{4}\left(\frac{r}{\sigma}\right)^{2}} .
$$

As a result,

$$
G_{R}(r)=\int_{0}^{r} g_{R}(u) d u=1-\exp \left[-\frac{1}{4}\left(\frac{r}{\sigma}\right)^{2}\right] .
$$

This is Eq. (1) in the text.

If $\sigma_{\mathbf{X}} \neq \sigma_{\mathbf{Y}}:$ then let

$$
\frac{1}{\sigma_{X}}=2 \sqrt{a+b} \text { and } \frac{1}{\sigma_{Y}}=2 \sqrt{a-b} .
$$

This leads to:

$$
\begin{aligned}
g_{R}(r) & =\frac{4 r \sqrt{a^{2}-b^{2}}}{\pi} \int_{0}^{\frac{\pi}{2}} e^{-r^{2}\left[(a+b) \cos ^{2} \theta+(a-b) \sin ^{2} \theta\right]} d \theta \\
& =\frac{4 \sqrt{a^{2}-b^{2}}}{\pi} r e^{-a r^{2}} \int_{0}^{\frac{\pi}{2}} e^{-b r^{2}\left(\cos ^{2} \theta-\sin ^{2} \theta\right)} d \theta \\
& =\frac{4 \sqrt{a^{2}-b^{2}}}{\pi} r e^{-a r^{2}} \int_{0}^{\frac{\pi}{2}} e^{-b r^{2} \cos 2 \theta} d \theta \\
& =\frac{2 \sqrt{a^{2}-b^{2}}}{\pi} r e^{-a r^{2}} \int_{0}^{\pi} e^{-b r^{2} \cos \alpha} d \alpha \\
& =2 \sqrt{a^{2}-b^{2}} r e^{-a r^{2}} I_{0}\left(b r^{2}\right) .
\end{aligned}
$$

where

$$
a=\frac{1}{8}\left(\frac{1}{\sigma_{X}^{2}}+\frac{1}{\sigma_{Y}^{2}}\right), \quad b=\frac{1}{8}\left(\frac{1}{\sigma_{X}^{2}}-\frac{1}{\sigma_{Y}^{2}}\right),
$$

and

$$
I_{0}(z)=\frac{1}{\pi} \int_{0}^{\pi} e^{-z \cos \alpha} d \alpha .
$$

is a modified Bessel function of the first kind [3, §9.6.16].

This result matches Eq. (5).

\section{The Edgeworth Approximation}

The Edgeworth approximation is a truncation of an infinite series that can closely approximate a wide class of functions. It is discussed in [7, pp. $17 \mathrm{ff}]$ to some extent and to a much greater degree in [8]. This development borrows some notation from [8], but does not go into as much detail as that presentation.

Let $\xi \triangleq \delta / \sigma_{\Delta_{X}}$. Note that $\mu_{\Xi}=0$ and $\sigma_{\Xi}=1$. Also,

$$
f_{\Delta_{X}}(\delta) d \delta=\frac{1}{\sigma_{\Delta_{X}}} f_{\Delta_{X}}(\xi) d \xi .
$$

In general ${ }^{3}$, we can expand the distribution of any density $f(\xi)$ into the series [8, Eq. (43)]

$$
f(\xi)=\left[1+\sum_{s=1}^{\infty} \sigma^{s} \mathcal{P}_{s}(\xi)\right] \phi(\xi),
$$

where $\mathcal{P}_{s}(\xi)$ is a $s+2$ order polynomial of $\xi$ with constant terms determined by the differences between first $s+2$ moments of $\Xi$ and the same moments of a standard normal. This series tends to converge rapidly, so the first few terms serve as a useful estimate of $f(\xi)$. The discussion in [7], for example, covers only to $s=2$.

In the case of $\Xi$, all odd moments are zero. Also, by setting $\sigma_{\Delta}=\sigma_{X} \sqrt{2}$, the difference between the variance of $\Xi$ and that of the normal approximation is also zero. Hence, the only non-zero term in a version of Eq. (44) truncated at $s=2$ will be for $s=2$. The approximation is, therefore,

$$
g_{\Delta}(\xi) \approx \frac{1}{\sigma_{\Delta_{X}}}\left[1+\frac{\gamma_{2_{\Delta_{X}}}}{24} H e_{4}(\xi)\right] \phi(\xi) .
$$

where

$$
\mathrm{He}_{4}(\xi)=\left(\xi^{4}-6 \xi^{2}+3\right)
$$

is a Chebyshev-Hermite polynomial ${ }^{4}$ of order 4 . This distribution has a kurtosis that matches that of $\Delta_{X}$, but all other moments match a $N\left(0, \sigma_{\Delta_{X}}\right)$ random variable. It is the basis of the $G_{R_{2}}$ approximation. The discussion in [8] explains how to add more terms. It also includes a computer program to help determine the higher-order coefficients.

\section{The $\mathrm{G}_{\mathrm{R}_{2}}$ Approximation}

The $G_{R_{2}}$ estimate is based on Eq. (45). Let

$$
\xi_{X}=\frac{\delta_{X}}{\sigma_{\Delta_{X}}}=\frac{\delta_{X}}{\sigma_{X} \sqrt{2}} \text { and } \xi_{Y}=\frac{\delta_{Y}}{\sigma_{\Delta_{X}}}=\frac{\delta_{Y}}{\sigma_{Y} \sqrt{2}} .
$$

Concentrating on $\xi_{X}$ for now, let

$$
\begin{aligned}
g_{\Delta_{X}}\left(\delta_{X}\right) & =\frac{1}{\sigma_{\Delta_{X}}}\left[1+\frac{\gamma_{2_{\Delta_{X}}}}{24} H e_{4}\left(\xi_{X}\right)\right] \phi\left(\xi_{X}\right) \\
& =\frac{1}{\sigma_{X} \sqrt{2}}\left[1+\frac{\gamma_{2_{X}}}{48} H e_{4}\left(\xi_{X}\right)\right] \phi\left(\xi_{X}\right) .
\end{aligned}
$$

\footnotetext{
${ }^{3}$ Here, we assume $\Xi$ is a continuous r.v. and $f(\xi)$ is both continuous and differentiable over the range of $\Xi$. See [8, p. 199] for analogous expressions in other cases.

${ }^{4}$ This is called the Hermite polynomial in [7].
} 
A similar argument leads to $g_{\Delta_{Y}}\left(\delta_{Y}\right)$. Assuming $X$ and $Y$ are independent, the joint distribution of $\delta_{X}$ and $\delta_{Y}$ is:

$$
\begin{aligned}
g_{\vec{\Delta}}\left(\delta_{X}, \delta_{Y}\right) & \\
= & \frac{1}{2 \sigma_{X} \sigma_{Y}}\left[1+\frac{\gamma_{2_{X}}}{48} H e_{4}\left(\xi_{X}\right)\right] \\
\quad & {\left[1+\frac{\gamma_{2_{Y}}}{48} H e_{4}\left(\xi_{Y}\right)\right] \varphi\left(\xi_{X}, \xi_{Y}\right) } \\
= & \frac{\varphi\left(\xi_{X}, \xi_{Y}\right)}{2 \sigma_{X} \sigma_{Y}} \\
\times\left[1+\frac{\gamma_{2_{X}}}{48}\right. & H e_{4}\left(\xi_{X}\right)+\frac{\gamma_{2_{Y}}}{48} H e_{4}\left(\xi_{Y}\right) \\
\quad+\frac{\gamma_{2_{X}}}{48} & \left.H e_{4}\left(\xi_{X}\right) \frac{\gamma_{2_{Y}}}{48} H_{e_{4}}\left(\xi_{Y}\right)\right] .
\end{aligned}
$$

Because the last term is of very low order, it is dropped. Thus,

$$
\begin{aligned}
g_{\vec{\Delta}_{2}}\left(\delta_{X}, \delta_{Y}\right) \triangleq \frac{\varphi\left(\xi_{X}, \xi_{Y}\right)}{2 \sigma_{X} \sigma_{Y}}[1 & +\frac{\gamma_{2_{X}}}{48} H e_{4}\left(\xi_{X}\right) \\
& \left.+\frac{\gamma_{2_{Y}}}{48} H e_{4}\left(\xi_{Y}\right)\right]
\end{aligned}
$$

is the basis for the $G_{R_{2}}$ estimate. Its first term is the density for the $G_{R_{1}}$ estimate. Let

$$
\begin{aligned}
g_{\vec{\Delta}_{2 b}}\left(\delta_{X}, \delta_{Y}\right) \triangleq & \frac{\varphi\left(\xi_{X}, \xi_{Y}\right)}{2 \sigma_{X} \sigma_{Y}} \\
& \times\left[\frac{\gamma_{2_{X}}}{48} H_{e_{4}}\left(\xi_{X}\right)+\frac{\gamma_{2_{Y}}}{48} H_{4}\left(\xi_{Y}\right)\right]
\end{aligned}
$$

be that portion of (48) that is not included in $G_{R_{1}}$. The only closed form solution presented here is when $\sigma_{X}=\sigma_{Y}=\sigma$ and $\gamma_{2_{X}}=\gamma_{2_{Y}}=\gamma_{2}$. In this case,

$$
\begin{aligned}
& g_{\vec{\Delta}_{2} b}\left(\delta_{X}, \delta_{Y}\right) \\
& \quad=\frac{\gamma_{2}}{96 \sigma^{2}}\left[H e_{4}\left(\xi_{X}\right)+H e_{4}\left(\xi_{Y}\right)\right] \varphi\left(\xi_{X}, \xi_{Y}\right) .
\end{aligned}
$$

Because $\sigma_{\Delta}=\sigma \sqrt{2}$, after the change of variables $\delta_{X}=$ $r \cos \theta$ and $\delta_{Y}=r \sin \theta$ the sum of the two ChebyshevHermite polynomials becomes:

$$
\left(\frac{r^{2}}{2 \sigma^{2}}\right)^{2}\left(\cos ^{4} \theta+\sin ^{4} \theta\right)-6\left(\frac{r^{2}}{2 \sigma^{2}}\right)+6
$$

Letting $u=[r /(2 \sigma)]^{2}$, Eq. (50) becomes:

$$
g_{\vec{\Delta}_{2 b}}(r, \theta)=\frac{\gamma_{2} r e^{-u}}{96 \pi \sigma^{2}}\left[2 u^{2}\left(\cos ^{4} \theta+\sin ^{4} \theta\right)-6 u+3\right] .
$$

This leads to:

$$
\begin{aligned}
g_{R_{2 b}}(r) & =4 \int_{0}^{\frac{\pi}{2}} g_{\vec{\Delta}_{2 b}}(r, \theta) d \theta \\
& =\frac{\gamma_{2} r}{32 \sigma^{2}} e^{-u}\left(u^{2}-4 u+2\right) .
\end{aligned}
$$

To obtain $G_{R_{2} b}(r)$, note that

$$
d u=\frac{r}{2 \sigma^{2}} d r .
$$

$$
\begin{aligned}
& \text { Thus, } \\
& \qquad \begin{aligned}
G_{R_{2} b}(r) & =\int_{0}^{r} g_{R_{2} b}(r) d r \\
& =\frac{\gamma_{2}}{16} \int_{0}^{\left(\frac{r}{2 \sigma}\right)^{2}}\left[u^{2}-4 u+2\right] e^{-u} d u \\
& =\frac{\gamma_{2}}{16}\left[-\left(\frac{r}{2 \sigma}\right)^{4}+2\left(\frac{r}{2 \sigma}\right)^{2}\right] e^{-\left(\frac{r}{2 \sigma}\right)^{2}} \\
& =\frac{\gamma_{2}}{256}\left[8\left(\frac{r}{\sigma}\right)^{2}-\left(\frac{r}{\sigma}\right)^{4}\right] e^{-\frac{1}{4}\left(\frac{r}{\sigma}\right)^{2}} .
\end{aligned}
\end{aligned}
$$

Adding this result to Eq. (1) leads to Eq. (3). 